\title{
Multiscale Curvature Assessment of Postural Deviations
}

\author{
Cornélia J.P. Passarinho ${ }^{1}$, Fátima N.S. Medeiros ${ }^{1}$, Jilseph Lopes Silva ${ }^{1}$, \\ Luís Henrique Cintra ${ }^{2}$, and Rafael B. Moreira ${ }^{1}$ \\ ${ }^{1}$ Federal University of Ceara \\ Campus do PICI, Bloco 705, C.P. 6007 \\ 60455-760 - Fortaleza, CE, Brazil \\ \{cjanayna, fsombra, jilseph, rafaelbarbosa\}@deti.ufc.br \\ http: //www.gpi.deti.ufc.br/index.html \\ ${ }^{2}$ Somma Physiotherapy Center \\ Rua Júlio Siqueira, 989, Dionísio Torres, CEP 60130-090 \\ Fortaleza-Ce, Brazil \\ Fax +55852728678 \\ lhcintra@secrel.com.br
}

\begin{abstract}
This paper presents and discusses an effective approach to assessing human postural deviations based on curvature measurements. Multiscale curvature values are calculated from both parametric contours extracted from the silhouette image in sagittal plane and from the medial axis. The algorithms were applied to digital images of patients who have been submitted to Global Postural Reeducation (GPR) physiotherapy treatment. Features such as area, perimeter, center of mass, spreading, thinness degree and angulations are also obtained for similarity shape analysis between images taken before and after the GPR treatment. The medial axis is evaluated to investigate how it can be used to infer the spine alignment of patients with postural deviations prior to taking exams such as x-ray or tomography.
\end{abstract}

\section{Introduction}

Unlike traditional physiotherapy approach, Global Postural Reeducation (GPR) physiotherapy treatment assesses human postural balance assuming human body as a complex set of interlinked segments. Thus, independent of the deviation's location, it is important to observe the entire human silhouette image. The images used in this methodology are taken at different planes by a digital camera. Images taken on sagittal plane provide knowledge of postural deviations such as thoracic kyphosis, lumbar hyperlordosis, cervical hyperlordosis, tibia angulation related to the feet, knee angulations (flexion and extension) and pelvis (anterior and posterior tilts) [1].

In this work the performance of the GPR treatment is evaluated according to a feasible curvature measure calculated to the sagittal plane contour of patient pictures that are taken before and after the treatment. This work adopts the multiscale curvature approach developed in [2]. 
Other approaches related to this paper include the method proposed by Koara et al. [3] that generates the contour model of each link element of an articulated object by using hierarchical part decomposition method (HPD). The HPD method can divide the body regions into characteristic elements without any model of link parameters or shape based on the distribution of high-curvature points of the object's contour in video sequences [3].

A new approach for object recognition based on polygonal approximation of the contour object was proposed in [4]. The authors adopted the polygon vertices as being the high curvature points of the contour applying the wavelet transform.

Chang and Liu [5] presented a modified version of the curvature scale space (CSS) image of the contour [6,7] to recognize hand posture.

The traditional clinical assessment of postural deviations in patients under physioteraphy treatment is achieved by the physiotherapist observing them in different image planes, including the profile one (sagittal plane) before, during and after the treatment. The patterns of normal postures [4] are used to guide the exam that includes head projection analysis, alignment of thorax and back, alignment of arms relative to trunk, flexion of the knees and lumbar region curvature of the patient. This clinical assessment depends on the specialist ability, specially for subtle cases. Thus, this work provides a novel assessment method that measures the contour curvature for both sagittal plane image contour (SPIC) and the external contour of the medial axis (CMA).

The procedure presented in this paper consists of two stages. In the first one, the curvature for both SPIC and CMA are calculated. In the second stage, the postural deviations are evaluated according to some features extracted from the patterns.

The remainder of this paper is organized as follows. Section 2 presents a brief description of the curvature method used to assess posture patterns. Section 3 describes the geometric features used to evaluate the human shapes. Section 4 presents the experimental results and Section 5 provides the concluding remarks.

\section{Background}

The curvature defines the orientation changes in the tangential direction in each point of a given curve. A plot of a boundary curvature function can reveal sharp peaks corresponding to convex or concave regions in the boundary. Thus, the curvature analysis can be used to assist the physiotherapist in detecting human postural deviations in sagittal plane images taken in the clinic according to some rules.

The most popular method for multiscale curvature estimation uses a series of Gaussian to convolve with the curve contour. The Gaussian standard deviation controls the smoothing effect and consequently the amount of details in the curve contour.

The representation of the original human contour is given in terms of the $x$ and $y$ coordinates along it. The signals $x$ and $y$ are obtained starting from the highest left point in the contour and following it in the counterclockwise manner. 
The parametrized form of a given regular curve $C$ is $C(t)=(x(t), \mathrm{y}(t))$, with $t \in \Gamma \subset$ $\Re$, i.e. the parameter $t$ has values over an interval $\Gamma$ of the real line $\Re$. The curvature of any point $C\left(t_{0}\right)=\left(x\left(t_{0}\right), \mathrm{y}\left(t_{0}\right)\right), t_{0} \in \Gamma$, is given by Equation (1). It measures direction changes on the contour and is defined as:

$$
k(t)=\frac{\dot{x}(t) \ddot{y}(t)-\ddot{x}(t) \dot{y(t)}}{\left[(\dot{x}(t))^{2}+(\dot{y}(t))^{2}\right]^{3 / 2}}
$$

where $\dot{x}(t), \dot{y}(t), \ddot{x}(t)$ and $\ddot{y}(t)$ are the first and the second order derivatives of $x$ and $y$ with respect to $t$.

The curvature of a sagittal plane image contour can be estimated in terms of the Fourier transform of its $x$ - and $y$ - signals. Multiplying a set of Gaussian functions, with distinct standard deviations, by the Fourier spectra of the parametric $x$ and $y$ signals of the contour yields a shrinkage effect on the original shape. To overcome this effect, a scalar coefficient $\Omega$ for energy correction is multiplied by the $x$ and $y$ signals, according to César Junior et al [3]. This scheme is used before applying Equation (1).

The proposed method consists in evaluating the curvature in each point of the patient's contour, searching for local maxima values, i.e. high curvature points (HCP). These values reflect the most significant changes in the posture balance due to the GPR treatment.

\subsection{Symmetry Analysis}

Skeletonization [8], also known as the medial axis transform, is a process for reducing foreground regions in a binary image to a skeleton that largely preserves the extent and connectivity of the original region while throws away most of the original foreground pixels. The medial axis transform (MAT) is particularly interesting in this paper to addressing posture issue since it is closely related to the feasible alignment of the human spine. This section presents the MAT, also called symmetry axis transform (SAT).

We have assumed the medial axis as being the symmetry axis for the sagittal plane image contour. We have observed that the medial axis reflects relevant changes in the posture balance and it can be used by the physiotherapist to infer the patient's posture.

The overlapping of the medial axes of the patients and their respective contours are displayed in Fig. 2. The patients present sagittal deviations that are reflected in their medial axes taken before the GPR treatment as show Figs. 2(a), 2(c) and 2(e)). These postures in Figs. 2(b), 2(d) and 2(f) were flattened by the GPR treatment. The patients exhibit a stretched silhouette and significant differences can be noted bycomparing the cervical and lumbar regions of the patients. 


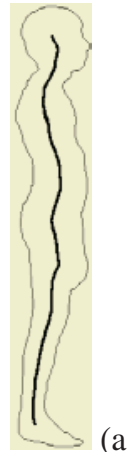

(a)

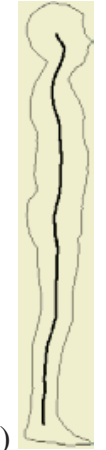

(b)

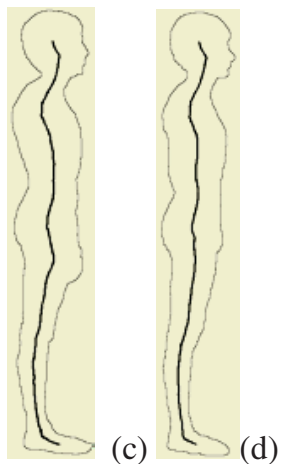

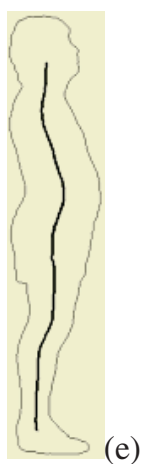

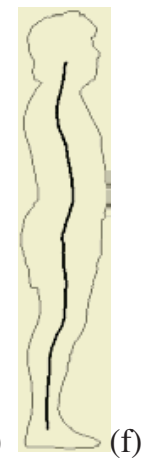

Fig. 1. The medial axis for sagittal plane image (bold line) of the (a) patient I before GPR, (b) patient I after GPR, (c) patient II before GPR, (d) patient II after GPR, (e) patient III before GPR and (f) patient III after GPR.

\section{Shape Analysis}

The aim of this section is to present features used for shape analysis. The idea consists in establishing the differences between the silhouette taken before and after the physiotherapy treatment. In the following, there are the relevant characteristics addressed to point out shape modifications due to the physiotherapy treatment.

In this approach, the uncalibrated area $(A)$ and perimeter $(P)$ in pixels represent the geometrical features that describe the human silhouette. Derived from them, the complexity $(C)$ achieves a small numerical value for simple geometrically silhouettes and larger values for complex ones. It is defined as:

$$
C=\frac{A}{2 \sqrt{\pi P}} .
$$

In [8] the area to perimeter ratio $(A P R)$ and thinness ratio $(T R)$ are described by equations (3) and (4), respectively.

$$
\begin{gathered}
A P R=\frac{A}{P} . \\
T R=4 \pi\left(\frac{A}{P^{2}}\right) .
\end{gathered}
$$

The center of mass (C.M.) can be used to show the stretching effect upon the patient posture. Derived from the center of mass, the maximum $\left(\mathrm{D}_{\max }\right)$ and minimum distances $\left(\mathrm{D}_{\min }\right)$ from the boundary points to the center of mass are useful to determine qualitatively the stretching degree of the posture. The spreading measure $(S)$ is derived from the principal component analysis [9], and it is presented in Equation (5) 
where $\lambda_{1}$ and $\lambda_{2}$ are the two eigenvalues associated with the covariance matrix and $\lambda_{1}>\lambda_{2}$. This measure will be low for long and thin silhouettes and high for flattened profiles.

$$
S=\frac{100 \lambda_{2}}{\lambda_{1}+\lambda_{2}} .
$$

\section{Experimental Results}

The images were taken by a digital camera in different planes in the beginning, in the middle and at the end of the GPR treatment. The selected patients wore shorts to reduced tactile hints from garments and stood barefoot on a fixed platform. Furthermore, they had short hair to avoid hiding the cervical spine. These procedures were taken to improve the assessment of the sagittal deviations.

Upon posture examination, patient I in Fig. 2(a) presents: lumbar hyperlordosis, thoracic hyperkiphosis and cervical hyperlordosis, head, hands and abdomen forward projected. After the GPR treatment, the new profile did not exhibit thoracic hyperkiphosis and the hands were aligned with the trunk. The thoracic and cervical regions presented standard flexion of the spine.

Relevant points in the sagittal plane image contour were marked as show Figs. 3(a) and 3(c) to call attention of the remarkable changes achieved in their surroundings by GPR. These points were selected because they were located in regions of the human contours that presented significant changes in the global postural balance, such as neck, abdomen, hands, thoracic kyphosis, lumbar lordosis and cervical lordosis. The modified posture of the patient I in Fig.3(c) due to the physiotherapy treatment was straightforwardly reflected in the curvature graphs of Figs. 3(d) and 3(g). The amplitude of the curvature values for these points displayed in Fig. 3(d) was decreased after GPR and the same effect was observed for the curvature (filled line) of the medial axis closed contour in Fig. 3(g).

By observing the curvature values and shape measures in Table 1 taken before and after GPR it can be noticed that all patients presented satisfactory results after the physiotherapy treatment. The curvature values for the points located in the cervical spine region confirmed the smoothing effect over the cervical hyperlordosis provided by the treatment. Similar results were observed in the lumbar spine, neck, abdomen, hands and thorax regions.

The shape measures in Table 1 pointed to an increasing tendency of the spreading measure, except for patient II, but an otherwise decreasing tendency of the thinness ratio for all patients. It implies that there was a stretching effect in the silhouette of the patients I, II and III derived from the physiotherapy treatment. The decreasing effect on the $x$ - coordinates of the center of mass indicates that the patient III did not present a compressed silhouette. It can be observed that the spinal curves were smoothed after GPR treatment. Even though the $x$ - coordinates for the other two patients did not decrease, the postural balance improvement became evident. 


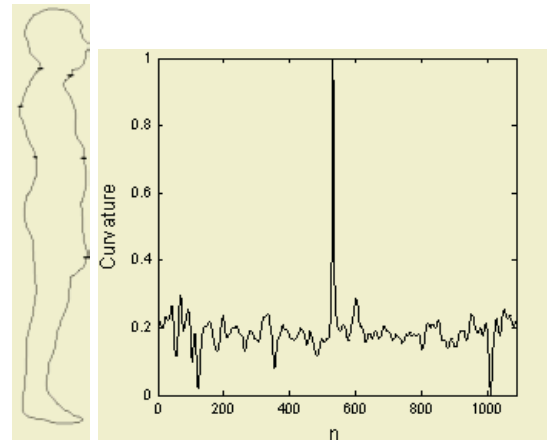

(a)

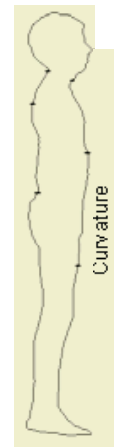

(b)

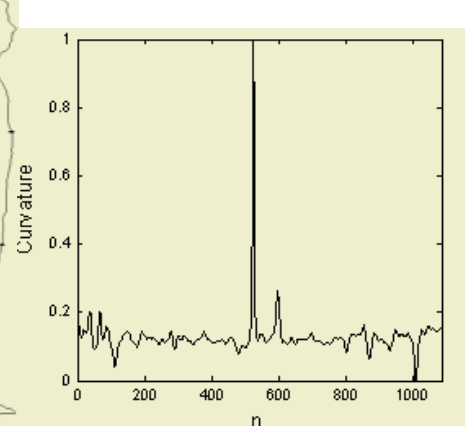

(c)

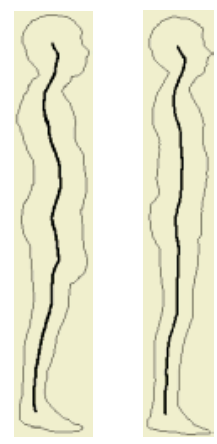

(e) (f)

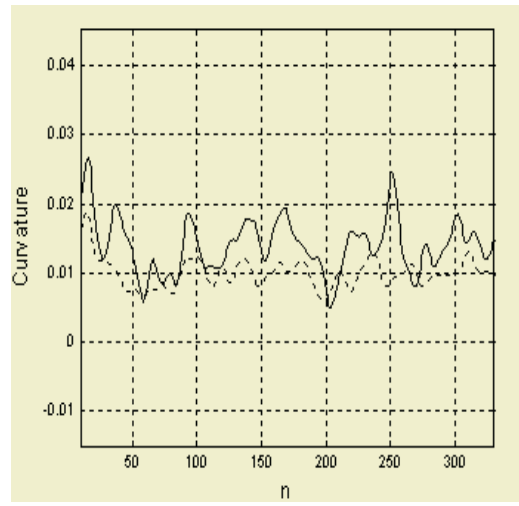

(g)

Fig. 2. Patient I and (a) its contour before GPR, (b) its respective curvature graph, (c) its contour after GPR (d) its respective curvature graph, (e) the medial axis (bold line) for the sagittal plane image before GPR, (f) the medial axis (bold line) for sagittal plane image after GPR and (g) a close detail of the overlapped curvatures calculated for the medial axis contour taken before (filled line) and after (dotted line) GPR.

\section{Concluding Remarks}

The smoothing effect over the curvatures caused by the GPR treatment was more straightforwardly visible for the contour curvature values related to lumbar and thoracic regions of the patients using this kind of physiotherapy. The external contour curvature of the medial axis was measured to substantiate the curvature results of the sagittal plane image contour and to infer the spine alignment. The remarkable changes on the postural balance of the patients pointed out to the benefits of the treatment. 
Table 1. Shape measures and curvature values of the patient's profiles taken before and after the GPR treatment.

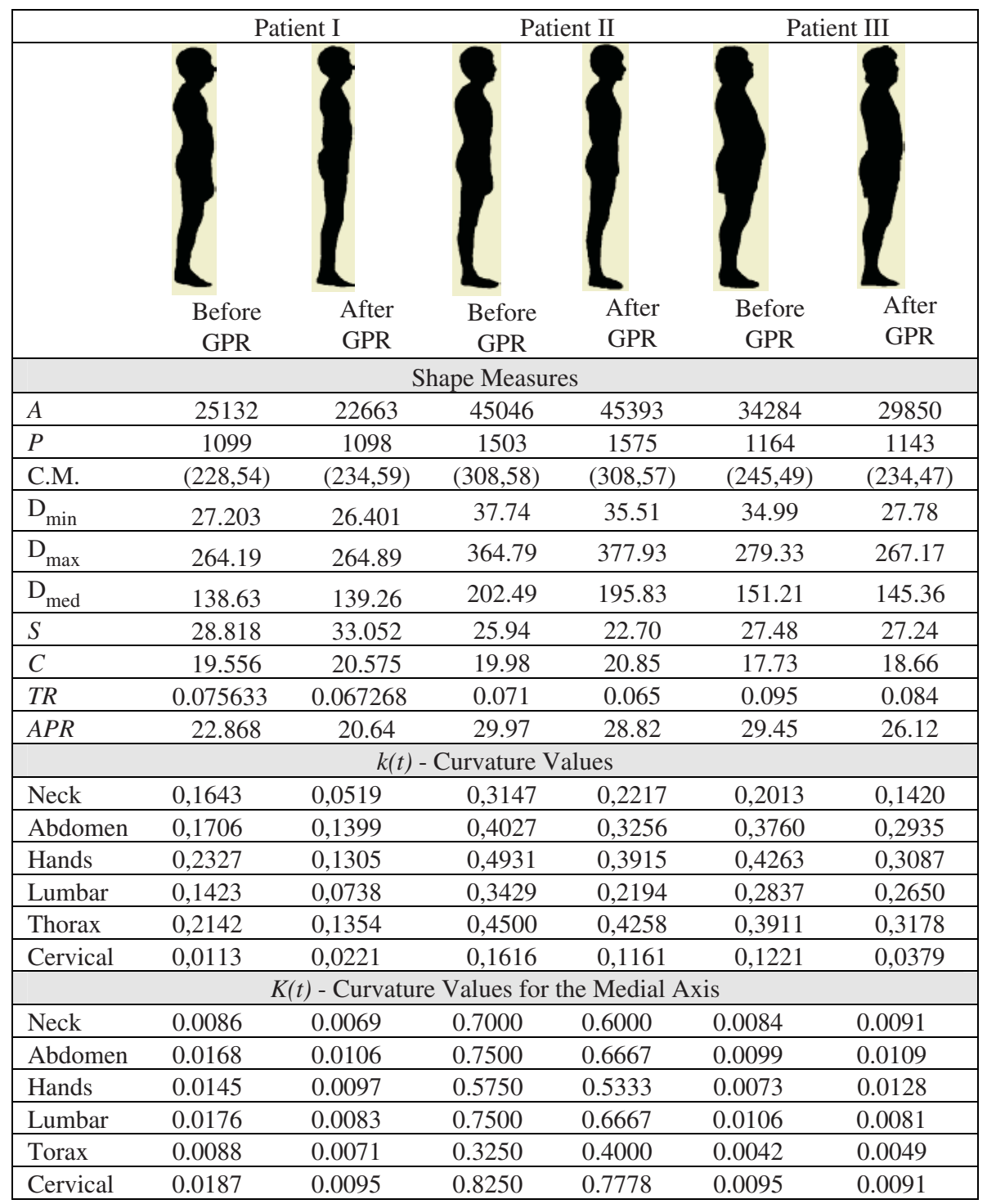

The proposed method seems to be a promising approach for automatic evaluation of posture deviations because it can support visual diagnosis performed by the physiotherapist. It reduces the subjective evaluation of postural deviations establishing a more precise clinical assessment tool. The advantage of this system over other medical imaging ones is the fact that it does not use ionizing radiation, it is portable, versatile, noninvasive and relatively low-cost. 


\section{Acknowledgment}

The authors are grateful to CAPES and CPNq for financial help.

\section{References}

1. Souchar, P.E.: Ginástica Postural Global. 2nd edn. Martins Fontes, São Paulo (1988)

2. César Junior, R. M., Costa, L. F.: Towards effective planar shape representation with multiscale digital curvature analysis based on signal processing techniques. Pattern Recognition. Vol. 29 (9) (1996) 1559-1569

3. Koara, K., Nishikawa, A., Miyazaki, F.: Hierarchical part decomposition method of articulated body contour, and its application to human body motion measurement. Proc. on IEEE/RSJ International Conference on Intelligent Robots and Systems. Vol. 3 Takamatsu Japan 31 October 5 November (2000) 2055 -2060

4. Cheikh, F., Quddus, A., Gabbouj, M.: Shape recognition based on wavelet-transform modulus maxima. The 7th IEEE International Conference on Electronics, Circuits and Systems. Vol. 1 Jounieh Lebanon December (2000) 461-464

5. Chang, C.C., Liu, C.: Modified curvature scale space feature alignment approach for hand posture recognition. Proc. on International Conference on Image Processing. Vol. 3 Barcelona September (2003) 309 -312

6. Mokhtarian, F., Mackworth, A.K.: Scale-based description and recognition of planar curves and two-dimensional shapes. IEEE Trans. on Pattern Analysis and Machine Intelligence. Vol. 8 (1) (1986) 34-43

7. Mokhtarian, F., Mackworth, A.K.: A theory of multiscale, curvature-based shape representation for planar curves. IEEE Trans. on Pattern Analysis and Machine Intelligence. Vol. 14 (8) (1992) 789-805

8. Costa, L.F., César Junior, R.M.: Shape Analysis and Classification: Theory and Practice. CRC Press USA 2001

9. Jollife, I. T.: Principal Component Analysis. Springer-Verlag New York 1986 